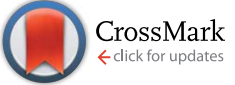

Cite this: RSC Adv., 2017, 7, 17289
Received 7th February 2017 Accepted 15th March 2017

DOI: 10.1039/c7ra01543g

rsc.li/rsc-advances

\section{Adamantyl derivatives and rearranged benzophenones from Garcinia xanthochymus fruits $\uparrow$}

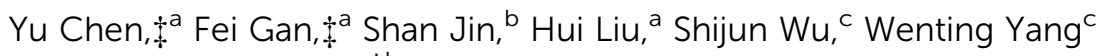 \\ and Guangzhong Yang*b
}

Garcinia xanthochymus is a tropical fruit yielding tree native to South East Asia. Its fruit is used to treat bilious conditions, diarrhea and dysentery. In this study, three new adamantyl derivatives, two new rearranged benzophenones, named as garcixanthochymones A-E (1-5), together with 12 known compounds including 7 xanthones (6-12) and 5 flavonoids (13-17) were isolated from the fruits of G. xanthochymus. Their structures were elucidated by detailed spectroscopic analyses. All isolated compounds were evaluated for their anti-proliferative activities against four human tumor cells (HepG2, A549, SGC7901, MCF-7). Adamantyl derivatives (1-3) and rearranged benzophenones (4-5) displayed potential inhibitory activity against four human cancer cell lines with $I C_{50}$ values of 5.16-16.45 $\mu \mathrm{M}$. These data suggested that the extracts of the fruits of $G$. xanthochymus are potent candidates for cancer prevention.

\section{Introduction}

Fruits contain high levels of biologically active components that impart health benefits beyond their basic nutritional value. Non-nutritive components in fruits such as phenolics, flavonoids, and in particular anthocyanins contribute in varying degrees to the antioxidant activity of individual fruits. Antioxidants play a crucial role in the prevention of chronic ailments such as heart attacks, cancer, diabetes, hypertension, stroke and Alzheimer's disease by combating oxidative stress. This suggests that a change in dietary behavior, such as increasing the consumption of fruits, may provide desirable health benefits for reducing the incidence of chronic diseases. ${ }^{1-3}$ The genus Garcinia belongs to Guttiferae (Clusiaceae) family and is a rich source of secondary metabolites including xanthones, flavonoids, benzophenones, lactones and phenolic acids. Several species have been documented as ethnobotanically important plants used traditionally as food and medicine. ${ }^{4}$ Garcinia xanthochymus is a fruit yielding tree native to South East Asia. Its fruits are edible and also used in jams preserves and vinegar. A preparation of the fruit of G. xanthochymus is given to treat

${ }^{a}$ College of Chemistry and Material Sciences, South-Central University for Nationalities, Wuhan 430074, P. R. China

${ }^{b}$ School of Pharmaceutical Sciences, South-Central University for Nationalities, Wuhan 430074, P. R. China. E-mail: yanggz888@126.com; Fax: +86-27-67841196; Tel: +8627-67841196

${ }^{c}$ College of Environmental and Biological Engineering, Wuhan Technology and Business University, Wuhan 430065, P. R. China

$\dagger$ Electronic supplementary information (ESI) available. See DOI: 10.1039/c7ra01543g

\$ These authors contributed equally to this work. bilious conditions, diarrhea and dysentery. ${ }^{5,6}$ Phytochemical studies have revealed that the fruits of G. xanthochymus contain various chemical constituents, including 7 polyisoprenylated benzophenones (PIBs): guttiferone $\mathrm{H}$, gambogenone, aristophenone A, xanthochymol, guttiferone E, cycloxanthochymol, isoxanthochymol, 4 xanthones: alloathyriol, maclurin, euxanthone, 1,5-dihydroxyxanthone and 8 bifavonoids: amentoflavone, 3,8"-biapigenin, ( \pm )-fukugetin, ( \pm )-fukugiside, 3,8" biflavanone, morelloflavone, GB 1a, $( \pm)$-volkensiflavone. ${ }^{6-9}$ The fruits of $G$. xanthochymus have been reported to show a variety of biological activities such as antioxidant, ${ }^{\mathbf{1 0}}$ antidiabetic, ${ }^{11}$ antiplasmodial ${ }^{7}$ and cytotoxic activities. ${ }^{12}$ It has been reported that the G. xanthochymus fruits possess $31.31 \mathrm{mg} \mathrm{g}^{-1}$ and $5.313 \mathrm{mg}$ $\mathrm{g}^{-1}$ of total phenol and flavonoid contents. ${ }^{2}$ PIBs are a major class of compounds isolated from the fruits of Garcinia plant, since fruits are the only edible plant part. PIBs with structural diversity have a wide range of biological activities. ${ }^{4}$ Much effort has been devoted to verify the effectiveness of the fruit of $G$. xanthochymus against cancer. A series of studies found that PIBs isolated from the fruit of G. xanthochymus showed inhibitory effects on human colon cancer cell lines by regulating the mammalian target of rapanmycin (mTOR) cell survival pathway. ${ }^{12}$

As part of our continuing research on bioactive components from nutritional fruits, we have investigated the fruits of $G$. xanthochymus, isolated and identified three new adamantyl derivatives, two new rearranged benzophenones, named as garcixanthochymones A-E (1-5) and 12 known compounds. Cytotoxicities against four human tumor cell lines (HepG2, A549, SGC7901, MCF-7) of the 17 isolated compounds are also reported. 


\section{Materials and methods}

\subsection{General experimental procedures}

Optical rotations were determined in $\mathrm{MeOH}$ on a P-1020 digital polarimeter. (JASCO Co., Tokyo, Japan). UV spectra were obtained on a 2401PC spectrophotometer (Shimadzu Co., Tokyo, Japan). 1D and 2D NMR spectra were recorded on a Bruker AVANCE III-500 MHz and a Bruker AV-400 MHz spectrometer (Bruker, Ettlingen, Germany) in DMSO- $d_{6}, \mathrm{C}_{5} \mathrm{D}_{5} \mathrm{~N}$, acetone- $d_{6}$ using tetramethylsilane (TMS) as an internal reference standard. Chemical shifts $(\delta)$ have been expressed in ppm and the coupling constants $(J)$ have been given in Hz. High-resolution electrospray mass spectroscopy was performed on an Agilent G6230 TOF mass spectrometer (HR-ESI-MS) (Agilent Technologies Co. Ltd., California, America) and A waters Autospec Premier 776 mass spectrometer (HR-EI-MS) (Waters Technologies Co. Ltd., Massachusetts, America). High-performance liquid chromatography (HPLC) was conducted on an Ultimate 3000 HPLC system (Dionex Co., Sunnyvale, CA, USA) equipped with an Ultimate 3000 pump and Ultimate 3000 variable wavelength detector, as well as a semi-preparative YMC-Pack ODS-A column $(250 \times 10 \mathrm{~mm}, 5 \mu \mathrm{m})$ and a preparative YMC-Pack ODSA column $(250 \times 20 \mathrm{~mm}, 5 \mu \mathrm{m})$ from YMC Co., Ltd (Kyoto, Japan), column chromatography (CC) was conducted over silica gel (200-300 mesh and 300-400 mesh, Qingdao Haiyang Chemical Industry Co., Ltd., Qingdao, China). Chromatographic grade acetonitrile was purchased from Chang Tech Enterprise Co., Ltd (Taiwan, China). The cell lines were purchased from the cell bank of Chinese Academy of Sciences (Shanghai, China). 3-(4,5-Dimethyl-2-thiazolyl)-2,5-diphenyl2- $H$-tetrazolium bromide (MTT) was purchased from Biosharp Co. Ltd. (Hefei, Anhui, China). Doxorubicin hydrochloride was purchased from Aladdin Chemical Co. Ltd. (Shanghai, China). Dulbecco modified Eagle medium (DMEM), phosphate buffer saline (PBS) and penicillin-streptomycin solution were purchased from Hyclone Laboratories, GE healthcare life science (Logan, UT, USA). Fetal bovine serum (FBS) was purchased from Gibco, Life technologies (Grand Island, NY, USA). Reagent grade dimethyl sulfoxide (DMSO) was purchased from Vetec, Sigma Chemical Co. (St. Louis, MO, USA). The absorbance was read on a Multiskan GO microplate reader (Thermo Fisher Scientific Inc. Waltham, MA, USA).

\subsection{Plant material}

The fruits of G. xanthochymus were purchased from Xishuangbanna Prefecture, Yunnan Province, P. R. China and identified by Prof. Ying-hong Zhao, Xishuangbanna Prefecture National Medicine Research Institute. The voucher specimen (2013 091 201) was deposited in the herbarium of School of Pharmaceutical Sciences, South Central University for Nationalities.

\subsection{Extraction and isolation}

The dried fruits of G. xanthochymus $(6.18 \mathrm{~kg}$ ) were powdered and extracted with $95 \% \mathrm{EtOH}$ at room temperature for three times (each time for $24 \mathrm{~h}$ ) to obtain EtOH extract $2.94 \mathrm{~kg}$, and then successively partitioned with petroleum ether (P. E.), EtOAc and $n$-BuOH to get petroleum ether extract $267 \mathrm{~g}$, EtOAc extract $711 \mathrm{~g}, n$-BuOH extract $460 \mathrm{~g}$. The EtOAc extract (332 g) was chromatographed on a silica gel column (200-300 mesh) eluted successively with P. E./EtOAc gradient (19:1, $9: 1,7: 3,6: 4$, $1: 1,4: 6,3: 7,0: 1$ ) to obtain 10 fractions (Fr. 1-Fr. 10). Fr. 3 (13.3 g) was subjected to octadecylsilane CC eluting with $\mathrm{H}_{2} \mathrm{O}$ $\mathrm{MeOH}(7: 3,6: 4,1: 1,4: 6,3: 7,2: 8,0: 1)$ to obtain 10 major fractions (Fr. 3.1-Fr. 3.10). Fr. $3.2(145.4 \mathrm{mg}$ ) was purified by semipreparative HPLC $\left(\mathrm{MeOH}-\mathrm{H}_{2} \mathrm{O}, 52: 48\right)$ to afford 11 (7.4 $\mathrm{mg})$ at $t_{\mathrm{R}} 14.0 \mathrm{~min}$. Fr. $3.4(33 \mathrm{mg})$ was purified by semipreparative HPLC $\left(\mathrm{MeOH}-\mathrm{H}_{2} \mathrm{O}, 55: 45\right)$ to afford $13(5.1 \mathrm{mg})$ at $t_{\mathrm{R}} 13.9 \mathrm{~min}$. Fr. 3.5 ( $287 \mathrm{mg}$ ) was purified by recrystallization to afford $10(6.5 \mathrm{mg})$, then further subjected to a silica gel column (300-400 mesh) eluted successively with $\mathrm{CHCl}_{3} / \mathrm{MeOH}$ gradient (200:1, $100: 1,50: 1,19: 1,7: 3,1: 1,3: 7,0: 1)$ to obtain 6 fractions (Fr. 3.5.1-Fr. 3.5.6). Fr. $3.5 .3(20 \mathrm{mg}$ ) was purified by semipreparative HPLC $\left(\mathrm{CH}_{3} \mathrm{CN}-\mathrm{H}_{2} \mathrm{O}, 35: 65\right)$ to afford 6 (1.5 $\mathrm{mg})$ at $t_{\mathrm{R}} 23.6 \mathrm{~min}$. Fr. $3.5 .4(10.5 \mathrm{mg})$ was purified by semipreparative HPLC $\left(\mathrm{MeOH}-\mathrm{H}_{2} \mathrm{O}, 50: 50\right)$ to afford $9(2.5 \mathrm{mg})$ at $t_{\mathrm{R}}$ $37.0 \mathrm{~min}$. Fr. 3.5 .5 (50 mg) was purified by semipreparative HPLC (MeOH- $\left.\mathrm{H}_{2} \mathrm{O}, 63: 37\right)$ to afford 7 (4.3 mg) at $t_{\mathrm{R}} 12.3 \mathrm{~min}, 8$ $(10.2 \mathrm{mg})$ at $t_{\mathrm{R}} 14.1 \mathrm{~min}$ respectively. Fr. 3.6 (1.16 g) was subjected to a silica gel column (300-400 mesh) eluted successively with $\mathrm{CHCl}_{3} / \mathrm{MeOH}$ gradient (200:1, $100: 1,50: 1,20: 1,10: 1$, $8: 2,6: 4,1: 1,2: 8,0: 1$ ) to obtain 7 fractions (Fr. 3.6.1-Fr. 3.6.7). Fr. 3.6.3 (350 mg) was purified by semipreparative HPLC with gradient $\mathrm{MeOH}-\mathrm{H}_{2} \mathrm{O}(73: 27$ to $98: 2)$ to afford 14 (23.1 $\mathrm{mg})$ at $t_{\mathrm{R}} 25.9 \mathrm{~min}$ and the lower polar fractions $(61.3 \mathrm{mg})$ at $t_{\mathrm{R}}$ $41 \mathrm{~min}$, which was further purified by semipreparative HPLC $\left(\mathrm{CH}_{3} \mathrm{CN}-\mathrm{H}_{2} \mathrm{O}, 64: 36\right)$ to afford $1(6.6 \mathrm{mg})$ at $t_{\mathrm{R}} 33.5 \mathrm{~min}, 2$ (4.1 $\mathrm{mg})$ at $t_{\mathrm{R}} 42.3 \mathrm{~min}, 3(4.0 \mathrm{mg})$ at $t_{\mathrm{R}} 37.4 \mathrm{~min}$. Fr. 3.7 (4.3 g) was subjected to a silica gel column (300-400 mesh) eluted successively with P. E./ $\mathrm{CHCl}_{3} / \mathrm{MeOH}$ gradient $(20: 5: 0.1,10: 5: 0.1$, $5: 5: 0.1,5: 5: 1,5: 5: 2,0: 9: 1,0: 0: 1)$ to obtain 3 fractions (Fr. 3.7.1-Fr. 3.7.3). Fr. 3.7 .3 (1.58 g) was subjected to a silica gel column (300-400 mesh) eluted successively with P. E. $/ \mathrm{CHCl}_{3} / \mathrm{MeOH}$ gradient (20: $5: 0.1,5: 5: 0.1,5: 5: 0.2$, $0: 0: 1$ ) to obtain 3 fractions (Fr. 3.7.3.1-Fr. 3.7.3.3). Fr. 3.7.3.2 $(620 \mathrm{mg})$ was purified by preparative $\mathrm{HPLC}\left(\mathrm{CH}_{3} \mathrm{CN}-\mathrm{H}_{2} \mathrm{O}, 93: 7\right)$ to obtain 11 fractions (Fr. 3.7.3.2.1-Fr. 3.7.3.2.11). Fr. 3.7.3.2.1 $(53.5 \mathrm{mg})$ was purified by semipreparative $\mathrm{HPLC}\left(\mathrm{CH}_{3} \mathrm{CN}-\mathrm{H}_{2} \mathrm{O}\right.$, $75: 25)$ to afford $4(23.2 \mathrm{mg})$ at $t_{\mathrm{R}} 19.5 \mathrm{~min}$. Fr. 3.7.3.2.2 (81.5 $\mathrm{mg})$ was purified by semipreparative HPLC $\left(\mathrm{CH}_{3} \mathrm{CN}-\mathrm{H}_{2} \mathrm{O}\right.$, $70: 30)$ to afford $5(24.9 \mathrm{mg})$ at $t_{\mathrm{R}} 31.1 \mathrm{~min}$. Fraction $5(9.5 \mathrm{~g})$ was subjected to octadecylsilane CC eluting with $\mathrm{H}_{2} \mathrm{O}-\mathrm{MeOH}(7: 3$, $6: 4,1: 1,4: 6,3: 7,2: 8,0: 1)$ to give 12 major fractions (Fr. 5.1-Fr. 5.12). Fr. 5.6 (30 mg) was purified by semipreparative HPLC $\left(\mathrm{MeOH}-\mathrm{H}_{2} \mathrm{O}, 82: 18\right)$ to afford $12(5.4 \mathrm{mg})$ at $t_{\mathrm{R}} 13.1 \mathrm{~min}$. Fraction 8 (49.9 g) was purified by recrystallization to afford 16 (364 mg). Fraction 7 (32.4 g) was subjected to a silica gel column (200-300 mesh) eluted successively with $\mathrm{CHCl}_{3} / \mathrm{MeOH}$ gradient (500:1, $100: 1,20: 1,10: 1,7: 3,0: 1$ ) to obtain 14 fractions (Fr. 7.1-Fr. 7.14). Fr. 7.12 (2.3 g) was subjected to octadecylsilane CC eluting with $\mathrm{H}_{2} \mathrm{O}-\mathrm{MeOH}(7: 3,1: 1,3: 7,1: 9,0: 1)$ to obtain 15 (200 mg) and 17 (166 mg). 
Garcixanthochymone A (1), white powder. $[\alpha]_{\mathrm{D}}^{21}=-13.8(c=$ 0.660, MeOH); UV (MeOH) $\lambda_{\max } \mathrm{nm}(\log \varepsilon): 258$ (4.07), 309 (3.79), 360 (3.86); ${ }^{1} \mathrm{H}-$ and ${ }^{13} \mathrm{C}-\mathrm{NMR}$ : see Table 1 . HR-EI-MS $m / z$ : $618.3550[\mathrm{M}]^{+}$(calcd for $\mathrm{C}_{38} \mathrm{H}_{50} \mathrm{O}_{7}$ : 618.3557).

Garcixanthochymone $\mathrm{B}(2)$, white powder. $[\alpha]_{\mathrm{D}}^{21}=-0.81(c=$ 0.410, MeOH); UV (MeOH) $\lambda_{\max } \mathrm{nm}(\log \varepsilon): 258$ (3.95), 310 (3.69), 363 (3.75); ${ }^{1} \mathrm{H}$ - and ${ }^{13} \mathrm{C}-\mathrm{NMR}$ : see Table 1. HR-ESI-MS (positive ion mode) $m / z$ : $639.3296[\mathrm{M}+\mathrm{Na}]^{+}$(calcd for $\mathrm{C}_{38} \mathrm{H}_{48} \mathrm{O}_{7} \mathrm{Na}$ : 639.3292).

Garcixanthochymone $\mathbf{C}(3)$, white powder. $[\alpha]_{\mathrm{D}}^{21}=+51.0(c=$ 0.400, MeOH); UV (MeOH) $\lambda_{\max } \mathrm{nm}(\log \varepsilon): 258$ (3.93), 309 (3.77), 357 (3.73); ${ }^{1} \mathrm{H}$ - and ${ }^{13} \mathrm{C}-\mathrm{NMR}$ : see Table 1 . HR-ESI-MS (positive ion mode) $m / z: 635.3577[\mathrm{M}+\mathrm{H}]^{+}$(calcd for $\mathrm{C}_{38} \mathrm{H}_{51} \mathrm{O}_{8}$ : 635.3578).

Garcixanthochymone $\mathbf{D}(4)$, white powder. $[\alpha]_{\mathrm{D}}^{21}=+9.49(c=$ 0.604, MeOH); UV (MeOH) $\lambda_{\max } \mathrm{nm}(\log \varepsilon): 260$ (3.95), 323 (3.67), 365 (3.77); ${ }^{1} \mathrm{H}$ - and ${ }^{13} \mathrm{C}-\mathrm{NMR}$ : see Table 2. HR-ESI-MS (positive ion mode) $m / z$ : $555.2718[\mathrm{M}+\mathrm{Na}]^{+}$(calcd for $\mathrm{C}_{33} \mathrm{H}_{40} \mathrm{O}_{6} \mathrm{Na}$ : 555.2723).

Garcixanthochymone $\mathbf{E}$ (5), white powder. $[\alpha]_{\mathrm{D}}^{21}=+22.7(c=$ 0.577, MeOH); UV (MeOH) $\lambda_{\max } \mathrm{nm}(\log \varepsilon): 260$ (3.97), 322 (3.71), 365 (3.77); ${ }^{1} \mathrm{H}$ - and ${ }^{13} \mathrm{C}-\mathrm{NMR}$ : see Table 2. HR-ESI-MS (positive ion mode) $m / z$ : $555.2726[\mathrm{M}+\mathrm{Na}]^{+}$(calcd for $\mathrm{C}_{33} \mathrm{H}_{40} \mathrm{O}_{6} \mathrm{Na}$ : $555.2723)$.

Table $1{ }^{1} \mathrm{H},{ }^{13} \mathrm{C}$ NMR data of compounds $1-3$ (in acetone- $\left.d_{6}\right)^{a}$

\begin{tabular}{|c|c|c|c|c|c|c|}
\hline \multirow[b]{2}{*}{ No. } & \multicolumn{3}{|c|}{${ }^{13} \mathrm{C}-\mathrm{NMR}$} & \multicolumn{3}{|l|}{${ }^{1} \mathrm{H}-\mathrm{NMR}$} \\
\hline & 1 & 2 & 3 & 1 & 2 & 3 \\
\hline 1 & 77.6 & 77.6 & 77.2 & & & \\
\hline 2 & 202.3 & 202.6 & 207.7 & & & \\
\hline 3 & 80.3 & 80.2 & 75.5 & & & \\
\hline 4 & 202.7 & 202.5 & 107.3 & & & \\
\hline 5 & 69.1 & 69.3 & 57.7 & & & \\
\hline 6 & 45.4 & 45.0 & 39.0 & $2.51 \mathrm{~m} 2 \mathrm{H}$ & $2.55 \mathrm{~m} 2 \mathrm{H}$ & $\begin{array}{l}3.63 \mathrm{dd}(14.8,4.8) \\
1.74 \mathrm{~m}\end{array}$ \\
\hline 7 & 48.3 & 48.3 & 43.4 & $1.81 \mathrm{~m}$ & $1.83 \mathrm{~m}$ & $2.23 \mathrm{~m}$ \\
\hline 8 & 54.5 & 54.4 & 57.0 & & & \\
\hline 9 & 204.3 & 204.3 & 208.0 & & & \\
\hline 10 & 192.3 & 192.1 & 198.2 & & & \\
\hline 11 & 128.9 & 128.6 & 133.2 & & & \\
\hline 12 & 116.8 & 117.4 & 119.5 & $6.91 \mathrm{~d}(2.0)$ & $6.87 \mathrm{~d}(1.6)$ & $7.12 \mathrm{~d}(2.0)$ \\
\hline 13 & 145.3 & 145.1 & 143.8 & & & \\
\hline 14 & 150.5 & 150.8 & 149.2 & & & \\
\hline 15 & 115.1 & 115.3 & 113.8 & $6.78 \mathrm{~d}(8.4)$ & $6.78 \mathrm{~m}$ & $6.70 \mathrm{~d}(8.4)$ \\
\hline 16 & 124.0 & 124.1 & 125.2 & $6.72 \mathrm{dd}(8.4,2.0)$ & $6.78 \mathrm{~m}$ & $6.97 \mathrm{dd}(8.4,2.0)$ \\
\hline \multirow[t]{2}{*}{17} & 34.9 & 34.4 & 28.8 & $1.89 \mathrm{dd}(14.4,6.0)$ & $1.91 \mathrm{dd}(14.8,6.8)$ & $2.17 \mathrm{dd}(13.2,3.2)$ \\
\hline & & & & $2.00 \mathrm{~m}$ & $1.98 \mathrm{dd}(14.8,6.8)$ & $1.30(\mathrm{~m})$ \\
\hline 18 & 43.8 & 39.9 & 41.7 & $2.73 \mathrm{~m}$ & $3.15 \mathrm{~m}$ & $1.56 \mathrm{~m}$ \\
\hline 19 & 149.4 & 149.0 & 80.1 & & & \\
\hline \multirow[t]{2}{*}{20} & 113.5 & 113.9 & 22.3 & $4.76 \mathrm{dd}(2.4,1.2)$ & $4.79 \mathrm{~d}(2.4)$ & $1.01 \mathrm{~s} 3 \mathrm{H}$ \\
\hline & & & & $4.66 \mathrm{~d}(2.4)$ & $4.83 \mathrm{~m}$ & \\
\hline 21 & 18.0 & 18.3 & 29.2 & $1.61 \mathrm{~s} 3 \mathrm{H}$ & $1.67 \mathrm{~s} 3 \mathrm{H}$ & $0.80 \mathrm{~s} 3 \mathrm{H}$ \\
\hline \multirow[t]{2}{*}{22} & 29.2 & 41.2 & 29.4 & $1.46 \mathrm{~m}$ & $1.62 \mathrm{~m}$ & $1.45 \mathrm{~m}$ \\
\hline & & & & $1.30 \mathrm{~m}$ & $1.37 \mathrm{~m}$ & $1.02 \mathrm{~m}$ \\
\hline 23 & 42.4 & 73.4 & 36.2 & $1.30 \mathrm{~m} 2 \mathrm{H}$ & $3.89 \mathrm{~d}(10.0)$ & $2.25 \mathrm{~m}, 2.02 \mathrm{~m}$ \\
\hline 24 & 70.5 & 150.1 & 146.7 & & & \\
\hline 25 & 29.5 & 109.7 & 110.7 & $1.17 \mathrm{~s} 3 \mathrm{H}$ & $4.72 \mathrm{~s} 4.93 \mathrm{~s}$ & $4.76 \mathrm{~s} 4.71 \mathrm{~s}$ \\
\hline 26 & 29.5 & 18.4 & 22.8 & $1.17 \mathrm{~s} 3 \mathrm{H}$ & $1.72 \mathrm{~s} 3 \mathrm{H}$ & $1.74 \mathrm{~s} 3 \mathrm{H}$ \\
\hline \multirow[t]{2}{*}{27} & 24.0 & 24.0 & 24.2 & 2.39 dd $(14.4,6.2)$ & 2.49 dd $(14.0,6.4)$ & $2.34 \mathrm{~m} 2 \mathrm{H}$ \\
\hline & & & & $2.50 \mathrm{~m}$ & $2.38 \mathrm{dd}(14.0,6.8)$ & \\
\hline 28 & 121.5 & 121.4 & 122.8 & $4.92 \mathrm{t}(6.2)$ & $4.89 \mathrm{t}(6.4)$ & $5.06 \mathrm{t}(7.0)$ \\
\hline 29 & 133.8 & 133.9 & 131.9 & & & \\
\hline 30 & 18.3 & 18.5 & 18.2 & $1.66 \mathrm{~s} 3 \mathrm{H}$ & $1.65 \mathrm{~s} 3 \mathrm{H}$ & $1.64 \mathrm{~s} 3 \mathrm{H}$ \\
\hline 31 & 26.1 & 26.2 & 26.2 & $1.60 \mathrm{~s} 3 \mathrm{H}$ & $1.58 \mathrm{~s} 3 \mathrm{H}$ & $1.64 \mathrm{~s} 3 \mathrm{H}$ \\
\hline 32 & 23.1 & 23.1 & 24.6 & $1.17 \mathrm{~s} 3 \mathrm{H}$ & $1.17 \mathrm{~s} 3 \mathrm{H}$ & $1.05 \mathrm{~s} 3 \mathrm{H}$ \\
\hline 33 & 22.7 & 22.7 & 23.5 & $1.22 \mathrm{~s} 3 \mathrm{H}$ & $1.22 \mathrm{~s} 3 \mathrm{H}$ & $1.02 \mathrm{~s} 3 \mathrm{H}$ \\
\hline 34 & 52.0 & 52.1 & 52.2 & $4.15 \mathrm{dt}(8.0,2.4)$ & $4.14 \mathrm{~d}(7.8)$ & $3.68 \mathrm{t}(3.2)$ \\
\hline 35 & 122.4 & 122.4 & 88.8 & $4.98 \mathrm{dt}(8.0,1.2)$ & $5.01 \mathrm{~d}(7.8)$ & $4.26 \mathrm{~d}(3.2)$ \\
\hline 36 & 134.2 & 134.2 & 71.7 & & & \\
\hline 37 & 26.3 & 26.2 & 29.5 & $1.69 \mathrm{~s} 3 \mathrm{H}$ & $1.69 \mathrm{~s} 3 \mathrm{H}$ & $1.34 \mathrm{~s} 3 \mathrm{H}$ \\
\hline 38 & 18.5 & 18.7 & 28.0 & $1.74 \mathrm{~s} 3 \mathrm{H}$ & $1.75 \mathrm{~s} 3 \mathrm{H}$ & $1.27 \mathrm{~s} 3 \mathrm{H}$ \\
\hline
\end{tabular}

${ }^{a}$ The chemical shifts have been expressed in $\delta$ ppm. The coupling constants $(J)$ have been expressed in Hz. 
Table $2{ }^{1} \mathrm{H},{ }^{13} \mathrm{C}$ NMR and $\mathrm{HMBC}$ data of compounds 4 and 5 (in $\mathrm{C}_{5} \mathrm{D}_{5} \mathrm{~N}$ )

\begin{tabular}{|c|c|c|c|c|c|c|}
\hline \multirow[b]{2}{*}{ No. } & \multicolumn{3}{|l|}{4} & \multicolumn{3}{|l|}{5} \\
\hline & ${ }^{13} \mathrm{C}-\mathrm{NMR}$ & ${ }^{1} \mathrm{H}-\mathrm{NMR}$ & HMBC & ${ }^{13} \mathrm{C}-\mathrm{NMR}$ & ${ }^{1} \mathrm{H}-\mathrm{NMR}$ & HMBC \\
\hline 1 & 64.4 & & & 64.3 & & \\
\hline 2 & 214.8 & & & 214.8 & & \\
\hline 3 & 71.2 & & & 71.2 & & \\
\hline 4 & 33.6 & $2.28,1.91 \mathrm{~m} 2 \mathrm{H}$ & & 33.8 & $1.97 \mathrm{dd}(11,8.5 \mathrm{~Hz}), 2.26 \mathrm{~m} 2 \mathrm{H}$ & \\
\hline 5 & 57.2 & $1.99 \mathrm{~m}$ & & 57.7 & $2.07 \mathrm{~m}$ & \\
\hline 6 & 42.8 & & & 42.7 & & \\
\hline 7 & 93.1 & & & 93.2 & & \\
\hline 8 & 70.1 & & & 70.1 & & \\
\hline 9 & 206.6 & & & 206.5 & & \\
\hline 10 & 48.3 & $1.91,2.10 \mathrm{~m} 2 \mathrm{H}$ & & 48.3 & $2.09 \mathrm{~m}, 1.91 \mathrm{~d}(13.5) 2 \mathrm{H}$ & \\
\hline 11 & 26.7 & $2.66 \mathrm{~m} 2 \mathrm{H}$ & $9,2,12,13,1,10$ & 26.7 & $2.66 \mathrm{~m} 2 \mathrm{H}$ & $9,2,12,13,1$ \\
\hline 12 & 120.8 & $5.54 \mathrm{t}(7.5)$ & & 120.6 & $5.54 \mathrm{t}(7.5)$ & 14,15 \\
\hline 13 & 134.2 & & & 134.2 & & \\
\hline 14 & 26.3 & $1.62 \mathrm{~s} 3 \mathrm{H}$ & 12,13 & 26.3 & $1.62 \mathrm{~s} 3 \mathrm{H}$ & 12,13 \\
\hline 15 & 18.3 & $1.63 \mathrm{~s} 3 \mathrm{H}$ & 12,13 & 18.3 & $1.64 \mathrm{~s} 3 \mathrm{H}$ & 12,13 \\
\hline 16 & 29.9 & $2.51 \mathrm{dd}(11.5,9.2), 2.24 \mathrm{~m} 2 \mathrm{H}$ & $18,17,3,2$ & 30.0 & $2.50 \mathrm{dd}(11.0,9.5), 2.23 \mathrm{~m} 2 \mathrm{H}$ & $18,17,3,2$ \\
\hline 17 & 57.6 & 3.07 dd $(9.2,8.5)$ & $7,8,18,19,20,9$ & 57.6 & 3.06 dd $(9.5,8.0)$ & $7,8,18,19,20,9$ \\
\hline 18 & 37.4 & & & 37.4 & & \\
\hline 19 & 27.7 & $1.24 \mathrm{~s} 3 \mathrm{H}$ & 17,29 & 27.8 & $1.22 \mathrm{~s} 3 \mathrm{H}$ & $17,29,18$ \\
\hline 20 & 31.0 & $1.42 \mathrm{~s} 3 \mathrm{H}$ & 17,29 & 31.0 & $1.39 \mathrm{~s} 3 \mathrm{H}$ & $17,29,18$ \\
\hline 21 & 33.8 & $1.84,1.91 \mathrm{~m} 2 \mathrm{H}$ & & 34.1 & $2.40 \mathrm{~m} 2 \mathrm{H}$ & \\
\hline 22 & 37.1 & $2.09,1.83 \mathrm{~m}$ & & 124.6 & $5.14 \mathrm{t}(7.0)$ & 24,25 \\
\hline 23 & 146.5 & & & 132.1 & & \\
\hline 24 & 110.7 & $4.78 \mathrm{~s}, 4.80 \mathrm{~s}$ & 25,22 & 26.3 & $1.68 \mathrm{~s} 3 \mathrm{H}$ & 22,23 \\
\hline 25 & 22.9 & $1.69 \mathrm{~s} 3 \mathrm{H}$ & 24,23 & 18.4 & $1.60 \mathrm{~s} 3 \mathrm{H}$ & 22,23 \\
\hline 26 & 19.7 & $1.78 \mathrm{~s} 3 \mathrm{H}$ & $7,6,10$ & 19.7 & $1.80 \mathrm{~s} 3 \mathrm{H}$ & $7,6,10,5$ \\
\hline 27 & 196.7 & & & 196.7 & & \\
\hline 28 & 129.0 & & & 129.0 & & \\
\hline 29 & 146.1 & & & 146.1 & & \\
\hline 30 & 112.4 & $7.23 \mathrm{~s}$ & $18,28,31,32$ & 112.4 & $7.22 \mathrm{~s}$ & $18,28,31,32$ \\
\hline 31 & 146.3 & & & 146.3 & & \\
\hline 32 & 154.0 & & & 154.0 & & \\
\hline 33 & 115.1 & $8.06 \mathrm{~s}$ & $27,32,31$ & 115.1 & $8.06 \mathrm{~s}$ & $27,32,31$ \\
\hline
\end{tabular}

\subsection{Measurement of inhibition activity on tumor cell proliferation}

The antiproliferative activities against four human tumor cell lines (HepG2, A549, SGC7901, MCF-7) of isolated compounds were measured by the MTT assay. The four human tumor cell lines were cultured in 96-well plates at a concentration of $10^{4}$ cells per well in DMEM containing 10\% FBS and 1\% penicillinstreptomycin at $37{ }^{\circ} \mathrm{C}$ in $5 \% \mathrm{CO}_{2}$ atmosphere. After $24 \mathrm{~h}$ of growth, washed the cells for three times by PBS, then added 200 $\mu \mathrm{L}$ sample solution or doxorubicin solution (dissolved in DMEM containing 1\% penicillin-streptomycin) in each well at a gradient of $50 \mu \mathrm{M}, 25 \mu \mathrm{M}, 12.5 \mu \mathrm{M}, 6.25 \mu \mathrm{M}$ and $3.125 \mu \mathrm{M}$. After $24 \mathrm{~h}$ of incubation, removed the cell culture medium and washed the cells for three times by PBS, then added $100 \mu \mathrm{L}$ MTT $\left(0.5 \mathrm{mg} \mathrm{mL}^{-1}\right.$ ) solution (dissolved in DMEM containing $1 \%$ penicillin-streptomycin) in each well incubated for $4 \mathrm{~h}$. After the incubation, removed the MTT solution and add $100 \mu \mathrm{L}$ DMSO, read the absorbance at a wavelength of $492 \mathrm{~nm}$ by using the microplate reader. The inhibition rate of the compound (\%) was calculated with the formula $[\mathrm{A}]_{\text {test }} /[\mathrm{A}]_{\text {control }} \times 100 \%$, where $[\mathrm{A}]_{\text {test }}$ is the absorbance of the test group that the cells incubated in sample solution, and $[\mathrm{A}]_{\text {control }}$ is the absorbance of the control group that the cells incubated only with the cell culture medium. The $\mathrm{IC}_{50}$ values was calculated according to the inhibition rate by using GraphPad Prism 6. All the 17 compounds have been tested the antiproliferative activities against four

Table 3 The antiproliferative activities of isolated compounds against four human tumor cells

\begin{tabular}{llrrr}
\hline$\left(\mathrm{IC}_{50}\right.$ in $\left.\mu \mathrm{M}\right)$ & \multicolumn{3}{l}{} \\
\hline Compounds $^{a}$ & \multicolumn{1}{l}{ HepG2 } & \multicolumn{1}{c}{ A549 } & \multicolumn{1}{c}{ SGC7901 } & \multicolumn{1}{c}{ MCF-7 } \\
\hline Doxorubicin $^{b}$ & $6.52 \pm 0.13$ & $14.03 \pm 0.21$ & $7.54 \pm 1.11$ & $4.40 \pm 1.17$ \\
$\mathbf{1}$ & $8.34 \pm 1.2$ & $15.88 \pm 0.48$ & $7.32 \pm 0.29$ & $5.77 \pm 0.17$ \\
$\mathbf{2}$ & $5.77 \pm 1.43$ & $15.13 \pm 5.99$ & $5.16 \pm 1.16$ & $14.12 \pm 1.29$ \\
$\mathbf{3}$ & $6.64 \pm 0.87$ & $16.25 \pm 3.11$ & $6.80 \pm 0.29$ & $9.75 \pm 0.62$ \\
$\mathbf{4}$ & $9.08 \pm 0.91$ & $7.48 \pm 0.03$ & $10.71 \pm 1.97$ & $15.69 \pm 0.87$ \\
$\mathbf{5}$ & $9.03 \pm 0.36$ & $8.24 \pm 0.16$ & $15.25 \pm 4.79$ & $16.45 \pm 0.23$ \\
$\mathbf{1 4}$ & $12.82 \pm 2.49$ & $11.29 \pm 1.40$ & $19.30 \pm 3.06$ & $13.55 \pm 1.97$
\end{tabular}

${ }^{a}$ Other isolates with $\mathrm{IC}_{50}>50 \mu \mathrm{M}$ for all cell lines are not listed. ${ }^{b}$ Doxorubicin was used as positive control. 
human tumor cell lines (HepG2, A549, SGC7901, MCF-7) as summarized in Table 3.

\section{Result and discussion}

\subsection{Structural elucidation of isolated compounds}

Garcixanthochymone A (1) was isolated as a white powder and gave the molecular formula $\mathrm{C}_{38} \mathrm{H}_{50} \mathrm{O}_{7}$ with 14 degrees of unsaturation by its HR-EI-MS ([M $]^{+} m / z$ 618.3550, calcd 618.3557). Its UV spectrum gave its maximum absorption wavelengths at $258 \mathrm{~nm}, 309 \mathrm{~nm}$ and $360 \mathrm{~nm}$ which indicated the presence of an aromatic moiety. The ${ }^{1} \mathrm{H}-\mathrm{NMR}$ spectrum revealed the presence of a 1,3,4-trisubstituted benzene ring signals $\left[\delta_{\mathrm{H}} 6.72(1 \mathrm{H}, \mathrm{dd}, J\right.$ $=8.4,2.0 \mathrm{~Hz}), 6.78(1 \mathrm{H}, \mathrm{d}, J=8.4 \mathrm{~Hz})$ and $6.91(1 \mathrm{H}, \mathrm{d}, J=2.0$ $\mathrm{Hz})]$, two olefinic proton signals $\left[\delta_{\mathrm{H}} 4.92(1 \mathrm{H}, \mathrm{t}, J=6.2 \mathrm{~Hz})\right.$ and $4.98(1 \mathrm{H}, \mathrm{dt}, J=8.0,1.2 \mathrm{~Hz})]$, one terminal double bonds $\left[\delta_{\mathrm{H}}\right.$ $4.66(1 \mathrm{H}, \mathrm{d}, J=2.4 \mathrm{~Hz})$ and $4.76(1 \mathrm{H}, \mathrm{dd}, J=2.4,1.2 \mathrm{~Hz})]$, nine methyl signals $\left[\delta_{\mathrm{H}} 1.17(3 \mathrm{H}, \mathrm{s}), 1.17(3 \mathrm{H}, \mathrm{s}), 1.17(3 \mathrm{H}, \mathrm{s}), 1.22\right.$ $(3 \mathrm{H}, \mathrm{s}), 1.60(3 \mathrm{H}, \mathrm{s}), 1.61(3 \mathrm{H}, \mathrm{s}), 1.66(3 \mathrm{H}, \mathrm{s}), 1.69(3 \mathrm{H}, \mathrm{s})$ and $1.74(3 \mathrm{H}, \mathrm{s})]$, one methylene signal $\left[\delta_{\mathrm{H}} 2.51(2 \mathrm{H}, \mathrm{m})\right]$, two methine signals $\left[\delta_{\mathrm{H}} 1.81(1 \mathrm{H}, \mathrm{m}), \delta_{\mathrm{H}} 4.15(1 \mathrm{H}, \mathrm{dt}, J=8.0,2.4\right.$ $\mathrm{Hz})]$. The ${ }^{13} \mathrm{C}-\mathrm{NMR}$ and DEPT spectrum indicated the presence of three nonconjugated carbonyl carbons $\left[\delta_{\mathrm{C}} 202.3,202.7\right.$, and 204.3], one conjugated carbonyl carbon $\left[\delta_{\mathrm{C}} 192.3\right]$, a 3,4-dihydroxybenzoyl group $\left[\delta_{\mathrm{C}} 115.1,116.8,124.0,128.9,145.3\right.$ and $150.5]$, two trisubstituted double bonds $\left[\delta_{\mathrm{C}} 121.5,122.4,133.8\right.$, and 134.2], one terminal double bonds $\left[\delta_{\mathrm{C}} 113.5,149.4\right]$, five quaternary carbons $\left[\delta_{\mathrm{C}} 54.5,69.1,70.5,77.6\right.$ and 80.3], five methylenes $\left[\delta_{\mathrm{C}} 24.0,29.2,34.9,42.4\right.$ and 45.4$]$, three methines $\left[\delta_{\mathrm{C}} 43.8,48.3\right.$ and 52.0], nine methyls $\left[\delta_{\mathrm{C}} 18.0,18.3,18.5,22.7\right.$, 23.1, 26.1, 26.3, 29.5 and 29.5]. On the basis of these data, 1 has an adamantyl phloroglucinol skeleton. ${ }^{13}$ Comparison of ${ }^{1} \mathrm{H}$ and ${ }^{13} \mathrm{C}$ NMR data of 1 with those of garcinialiptone $\mathrm{A}^{\mathbf{1 4}}$ indicated that the two compounds were closely related, except for a 3- hydroxy-3-methylbutyl group at C-18 of $1\left[\delta_{\mathrm{H}} 1.17(6 \mathrm{H}, \mathrm{s}), \delta_{\mathrm{H}} 1.30\right.$ $(3 \mathrm{H}, \mathrm{m}), \delta_{\mathrm{H}} 1.46(1 \mathrm{H}, \mathrm{m}) ; \delta_{\mathrm{C}} 29.2(\mathrm{C}-22), 29.5(\mathrm{C}-25,26), 42.4(\mathrm{C}-$ 23), 70.5 (C-24)] instead of a 3-methylbut-3-enyl group at C-18 of garcinialiptone A. ${ }^{\mathbf{1 4}}$ This assumption was further confirmed by the ${ }^{1} \mathrm{H}-{ }^{1} \mathrm{H}$ COSY form $\mathrm{H}-18$ to $\mathrm{H}_{2}-22$ and $\mathrm{H}_{2}-22$ to $\mathrm{H}_{2}-23$ and HMBC correlations (see Fig. 2) from $\mathrm{H}_{3}-25, \mathrm{H}_{3}-26$ to $\mathrm{C}-23\left(\delta_{\mathrm{C}}\right.$ 42.4) and C-24 $\left(\delta_{\mathrm{C}} 70.5\right)$. Regarding the relative configuration of 1, the ROESY spectrum of $\mathbf{1}$ (see Fig. 3) showed correlations of $\mathrm{H}-34 / \mathrm{H}_{3}-33, \mathrm{H}-34 / \mathrm{H}-7$, which indicated that $\mathrm{H}-34, \mathrm{H}-7, \mathrm{H}_{3}-33$ are on the same side of molecular plane which was the same as that of garcinialiptone A. Furthermore, the ROESY correlations between $\mathrm{H}-35 / \mathrm{H}_{2}-6, \mathrm{H}_{2}-6 / \mathrm{H}_{3}-32$ revealed that the $\mathrm{H}-35, \mathrm{H}_{3}-32$ and $\mathrm{H}_{2}-6$ were on the opposite face to $\mathrm{H}-34, \mathrm{H}-7, \mathrm{H}_{3}-33$. As a result of the above information, the structure of 1 was deduced completely as showed in the Fig. 1.

Garcixanthochymone B (2) was obtained as a white powder and gave the molecular formula $\mathrm{C}_{38} \mathrm{H}_{48} \mathrm{O}_{7}$ with 15 degrees of unsaturation by its HR-ESI-MS $\left([\mathrm{M}+\mathrm{Na}]^{+} \mathrm{m} / z\right.$ 639.3296, calcd 639.3292). Both 2 and 1 gave similar ${ }^{1} \mathrm{H}$ and ${ }^{13} \mathrm{C}$ NMR spectra data, except for the signals of a 2-hydroxy-3-methylbut-3-enyl group $(\mathrm{C}(22)-\mathrm{C}(26))$ at $\mathrm{C}-18$ in the former rather than 3hydroxy-3-methylbutyl group in the latter. This finding was also supported by the ${ }^{13} \mathrm{C}$ NMR data of C-23 ( $\delta$ 73.4), C-24 ( $\delta$ 150.1), and C-25 ( $\delta$ 109.7) and HMBC correlations (see Fig. 2$)$ between H-23/C-26, 22, 25, 24. Thus, compound 2 was elucidated as 23hydroxy garcinialiptone $\mathrm{A}$, named as garcixanthochymone $\mathrm{B}$.

Garcixanthochymone C (3) was obtained as a white powder and gave the molecular formula $\mathrm{C}_{38} \mathrm{H}_{50} \mathrm{O}_{8}$ with 14 degrees of unsaturation by its HR-ESI-MS $\left([\mathrm{M}+\mathrm{H}]^{+} \mathrm{m} / \mathrm{z}\right.$ 635.3577, calcd 635.3578). In comparison of the NMR data of 3 with those of 1 , revealed that 3 contained an adamantyl phloroglucinol skeleton as that of 1 , but lacked resonances for an olefinic proton in ${ }^{1} \mathrm{H}$ NMR and one trisubstituted double bond in ${ }^{13} \mathrm{C}$ NMR in 1. In contrast, four additional carbon resonances, including one
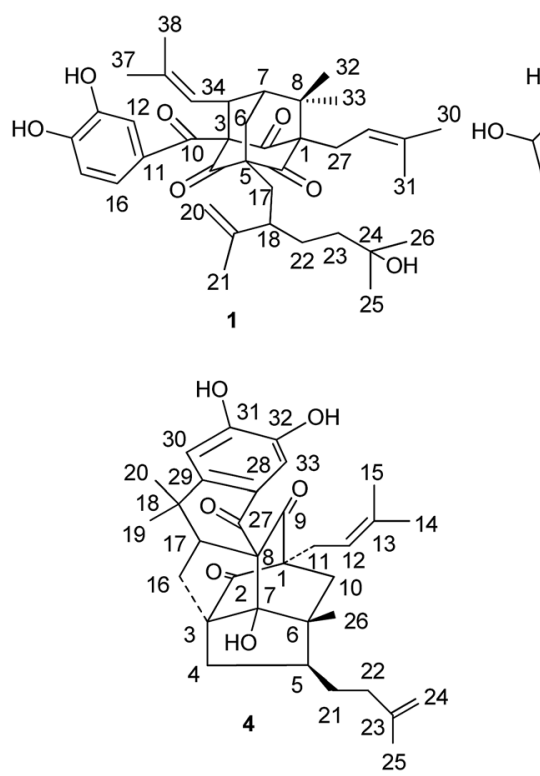

Fig. 1 Structures of compounds 1-5. 

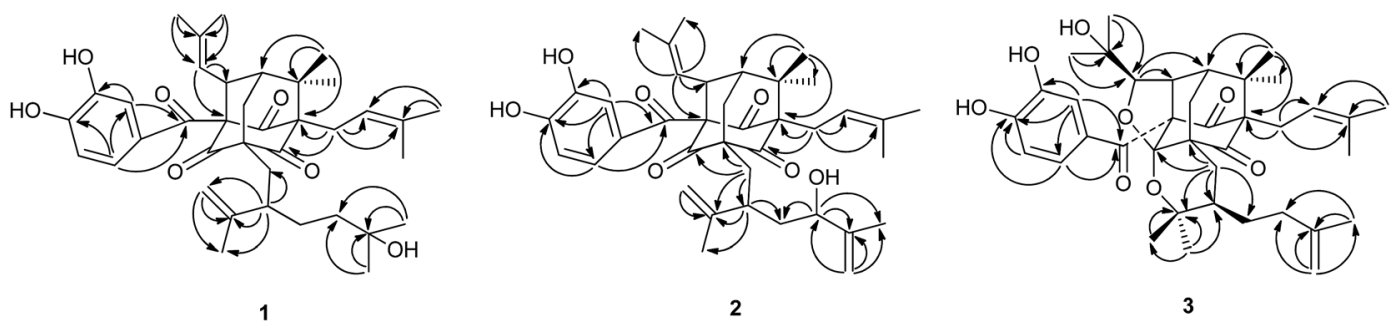

Fig. 2 Selected HMBC for compound 1-3.

oxygenated methine $\left(\delta_{\mathrm{C}} 88.8\right)$, two oxygenated quaternary carbon $\left(\delta_{\mathrm{C}} 71.7,80.1\right)$ and one ketal carbon $\left(\delta_{\mathrm{C}} 107.3\right)$ were found in 3. It suggested that the 2-methyl-2-propenyl group at C34 and 2-isopropenyl-5-methyl-5-hydroxyhexyl group at C-5 in $\mathbf{1}$ had undergone an alternative cyclization. HMBC correlations (see Fig. 2) of $\delta_{\mathrm{H}} 1.27\left(\mathrm{CH}_{3}-37\right)$ and $1.34\left(\mathrm{CH}_{3}-38\right)$ to the carbons at $\delta_{\mathrm{C}} 88.8(\mathrm{C}-35)$ and $71.7(\mathrm{C}-36)$, of $\delta_{\mathrm{H}} 4.26(\mathrm{H}-35)$ to the carbons at $\delta_{\mathrm{C}} 52.2(\mathrm{C}-34)$ and $43.4(\mathrm{C}-7)$ indicated that a 2,3-dioxygenated 2-methylpropyl group was attached to C-34 instead of 2-methyl2-propenyl group at C-34 in 1. HMBC correlations of $\delta_{\mathrm{H}} 1.01$ $\left(\mathrm{CH}_{3}-20\right)$ and $0.80\left(\mathrm{CH}_{3}-21\right)$ to the carbons at $\delta_{\mathrm{C}} 80.1(\mathrm{C}-19)$ and 41.7 (C-18) indicated that 2-oxygenated isopropyl group was attached to C-18 instead of isopropenyl group at C-18 in 1. The aforementioned groups accounted for 12 out of 14 degrees of unsaturation in compound 3 , indicating the presence of two additional rings. The chemical shifts of C-35 $\left(\delta_{\mathrm{C}} 88.8\right)$ and C-19 $\left(\delta_{\mathrm{C}} 80.1\right)$ and one more ketal carbon $\left(\delta_{\mathrm{C}} 107.3\right)$ implied the ether ring closure between C-35 and C-4, C-19 and C-4 to construct 2(1-hydroxy-1-methylethyl)-tetrahydrofuran ring fused with the phloroglucinol moiety at C-3 and C-4 and 2,2-dimethyl-3-(3methylbut-3-enyl)-tetrahydropyrano ring fused with the phloroglucinol moiety at C-5 and C-4. Regarding the relative configuration of 3, the ROESY spectrum of 3 (see Fig. 3) showed correlations of $\mathrm{H}-34 / \mathrm{H}_{3}-33, \mathrm{H}-34 / \mathrm{H}-7, \mathrm{H}-34 / \mathrm{H}-35, \mathrm{H}_{2}-6 \mathrm{a} / \mathrm{H}_{3}-32$ and $\mathrm{H}_{2}-6 \mathrm{~b} / \mathrm{H}_{3}-37$ which indicated that $\mathrm{H}-34, \mathrm{H}-35, \mathrm{H}-7$ and $\mathrm{H}_{3}-$ 33 are on the same side of molecular plane and $\mathrm{H}_{2}-6$ and $\mathrm{H}_{3}-32$ are on the other side of molecular plane. The ROESY correlations between Heq- 17 at $\delta_{\mathrm{H}} 2.17(1 \mathrm{H}, \mathrm{dd}, J=13.2,3.2) / \mathrm{H}-18$ and $\mathrm{H}-18 / \mathrm{H}_{3}-21$ revealed that the $\mathrm{C}-18$ side chain on the tetrahydropyran ring should be in an equatorial configuration. Therefore, the structure of $\mathbf{3}$ was deduced completely as showed in the Fig. 1.
Garcixanthochymone D (4) was obtained as a white powder and gave the molecular formula $\mathrm{C}_{33} \mathrm{H}_{40} \mathrm{O}_{6}$ with 14 degrees of unsaturation by its HR-ESI-MS $\left([\mathrm{M}+\mathrm{Na}]^{+} \mathrm{m} / z\right.$ 555.2718, calcd 555.2723). The ${ }^{1} \mathrm{H}$ NMR spectrum exhibited three olefinic protons $\left[\delta_{\mathrm{H}} 4.78(1 \mathrm{H}, \mathrm{s}), 4.80(1 \mathrm{H}, \mathrm{s}), 5.54(1 \mathrm{H}, \mathrm{t}, J=7.5 \mathrm{~Hz})\right]$, a 1,2,4,5-tetrasubstituted benzene moiety $\left[\delta_{\mathrm{H}} 7.23(1 \mathrm{H}, \mathrm{s}), 8.06\right.$ $(1 \mathrm{H}, \mathrm{s})]$, and six singlet methyls $\left[\delta_{\mathrm{H}} 1.24(3 \mathrm{H}, \mathrm{s}), 1.42(3 \mathrm{H}, \mathrm{s}), 1.62\right.$ $(3 \mathrm{H}, \mathrm{s}), 1.63(3 \mathrm{H}, \mathrm{s}), 1.69(3 \mathrm{H}, \mathrm{s}), 1.78(3 \mathrm{H}, \mathrm{s})]$. The ${ }^{13} \mathrm{C}$ NMR spectrum showed 33 carbons attributable to six methyls, seven methylenes, of which one is a terminal olefinic carbon $\left[\delta_{\mathrm{C}} 110.7\right.$ (C-24)], five methines, of which two are aromatic and one olefinic [ $\delta_{\mathrm{C}} 112.4$ (C-30), 115.1 (C-33), 120.8 (C-12)], 15 quaternary carbons, of which three are keto groups $\left[\delta_{\mathrm{C}} 196.7\right.$ (C-27), 206.6 (C-9), 214.8 (C-2)]. From the above analysis of the ${ }^{1} \mathrm{H}$ and ${ }^{13} \mathrm{C}$-NMR spectroscopic data, it was implied that compound 4 is a tetracyclo $\left[4 \cdot 3 \cdot 2.1^{1,6} \cdot 0^{3,7}\right]$ decane derivative similar to that of doitunggarcinone A. $^{15}$ Further comparison of the NMR spectroscopic data of $\mathbf{4}$ with those of doitunggarcinone A revealed that a 1,2,4,5-tetrasubstituted benzene moiety fused at C-28 and C-29 in 4 instead of an ortho-disubstituted benzene moiety in doitunggarcinone $\mathrm{A}$. This difference was evidenced by the HMBC correlations from $\mathrm{H}-30\left(\delta_{\mathrm{H}} 7.23\right)$ to $\mathrm{C}-18\left(\delta_{\mathrm{C}} 37.4\right)$ and $\mathrm{C}-28\left(\delta_{\mathrm{C}} 129.0\right)$ and from $\mathrm{H}-33\left(\delta_{\mathrm{H}} 8.06\right)$ to $\mathrm{C}-27\left(\delta_{\mathrm{C}} 196.7\right)$. The relative configuration of doitunggarcinone $\mathrm{A}$ was previously deduced from ROESY correlations, and 3-methylbut-3-enyl at C5 was determined as $\alpha$-orientation which was opposite to $\mathrm{CH}_{3}-$ 26 configuration. However, the relative stereochemistry of doitunggarcinone A have been revised by synthesis and comparison of the ${ }^{13} \mathrm{C}$ NMR spectral data with those of garcibracteatone and 5-epi-garcibracteatone. It was suggested that the stereochemistry at C-5 is a $\beta$-orientation. ${ }^{16}$ In order to determine the relative stereochemistry of $4,{ }^{1} \mathrm{H},{ }^{13} \mathrm{C}-\mathrm{NMR}$ and $2 \mathrm{D}$-NMR of 4

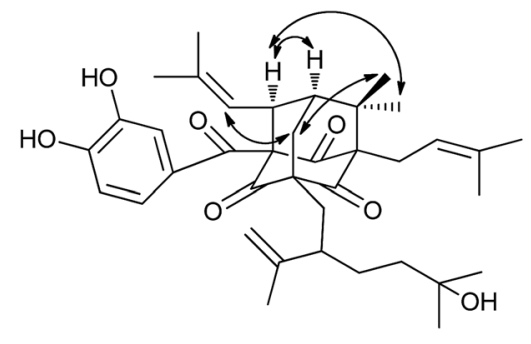

1

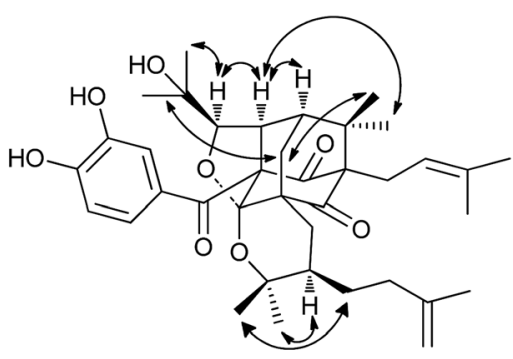

3

Fig. 3 Selected ROESY of compounds 1 and 3. 


.

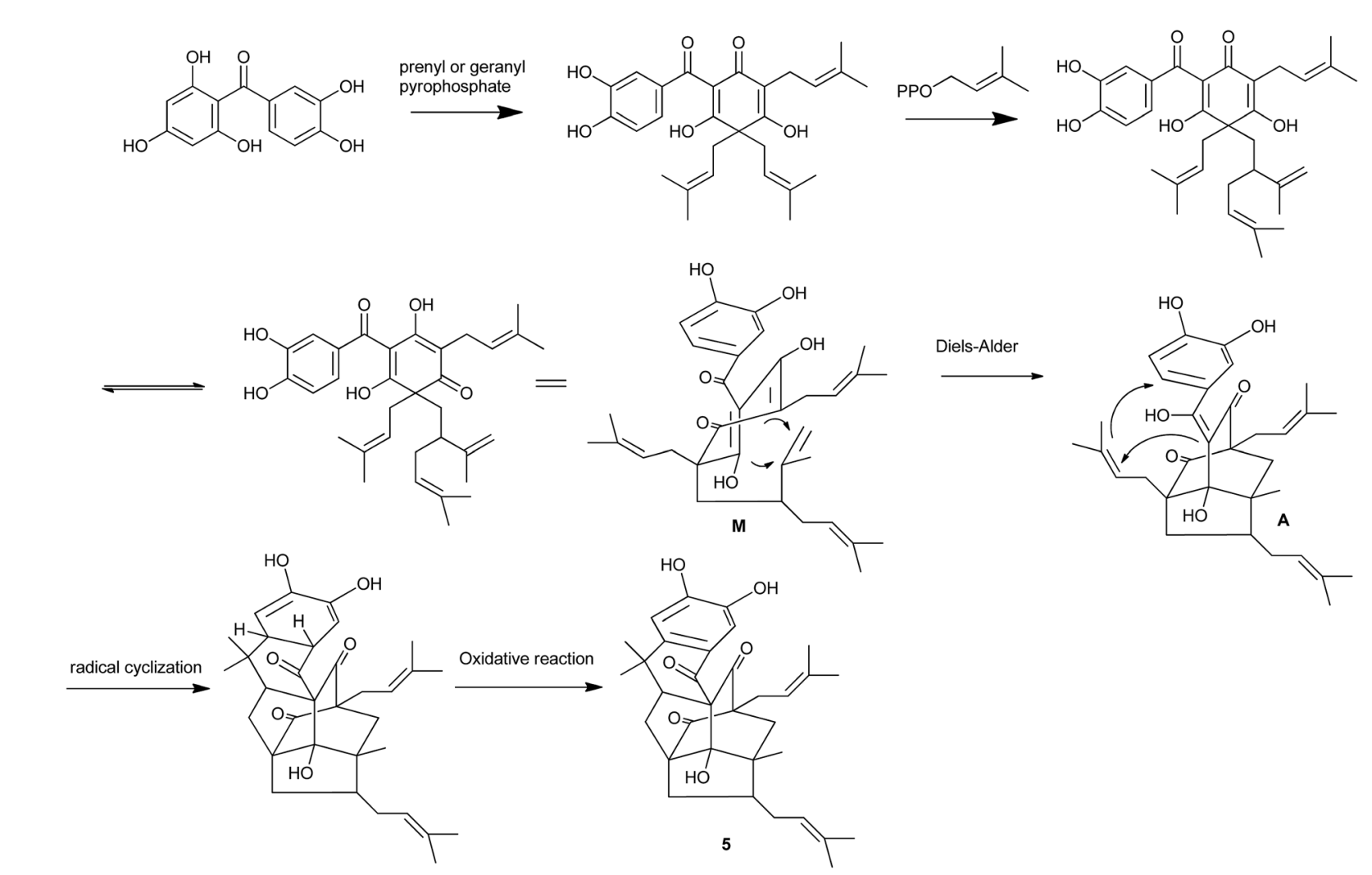

Scheme 1 Plausible biogenetic pathway for 5.

were measured again in $\mathrm{CDCl}_{3}$. By comparison of the ${ }^{13} \mathrm{C} \mathrm{NMR}$ spectral data of $\mathbf{4}$ with those of doitunggarcinone $\mathrm{A}$ in $\mathrm{CDCl}_{3}$, it suggested that the relative stereochemistry of $\mathbf{4}$ is the same as that of doitunggarcinone A. Thus, the structure of 4 was deduced completely as showed in the Fig. 1.

Garcixanthochymone E (5) was obtained as a white powder and gave the molecular formula $\mathrm{C}_{33} \mathrm{H}_{40} \mathrm{O}_{6}$ with 14 degrees of unsaturation by its HR-ESI-MS $\left([\mathrm{M}+\mathrm{Na}]^{+} \mathrm{m} / z\right.$ 555.2726, calcd 555.2723 ), which was the same as that of 4 . By comparing the NMR data of $\mathbf{5}$ with those of $\mathbf{4}$, it indicated that $\mathbf{5}$ was the isomer of 4 . However, the main difference found was that compound 5 displays ${ }^{1} \mathrm{H}$ NMR and ${ }^{13} \mathrm{C}$ NMR data for a 3-methylbut-2-enyl group at $\mathrm{C}-5$ instead of a 3-methylbut-3-enyl group in 4. This deduction was supported by ${ }^{1} \mathrm{H}^{-1} \mathrm{H}$ COSY correlations of $\mathrm{H}_{2}-4$ / $\mathrm{H}-5 / \mathrm{H}_{2}-21 / \mathrm{H}-22$. Thus, the structure of 5 was determined and named garcixanthochymone $\mathrm{E}$.

Polycyclic polyprenylated acylphloroglucinols (PPAPs) are a class of secondary metabolites that usually possess bicyclo [3.3.1]nonane-2,4,9-trione, adamantyl, or homoadamantyl-like core structures. ${ }^{17}$ Up to now, 7 PPAPs have been isolated and identified from G. xanthochymus, such as xanthochymol and isoxanthochymol which belongs to bicycle [3.3.1] nonane-2,4,9trione type. ${ }^{6}$ To the best of our knowledge, it was firstly time to isolate adamantly PPAPs from this plant. In addition, compound 3 featured two additional furan and pyrano rings incorporated into the tricyclo [3.3.1.1 $\left.1^{3,7}\right]$ decane system which are unique in natural adamantyl PPAPs. Few adamantyl-like PPAPs, such as sampsoniones $\mathrm{A}, \mathrm{B},{ }^{18}$ hyperisampsins $\mathrm{D}^{\mathbf{1 9}}$ and xerophenones $\mathrm{A}$ and $\mathrm{B}^{\mathbf{2 0}}$ were found to possess a hemiketal carbon in the core skeleton, therefore, 3 represents the first example of adamantyl PPAPs with an unusual ketal carbon in the adamantyl skeleton.

Structurally, compound $\mathbf{4}$ and $\mathbf{5}$ was elucidated to possess an unprecedented tetracyclo-[4.3.2.1 $\left.{ }^{1,6} \cdot 0^{3,7}\right]$ decane core similar to that of garcibracteatone, ${ }^{21}$ doitunggarcinone $\mathrm{A},{ }^{15}$ hyperuralone $\mathrm{B},{ }^{22}$ hyphenrone $\mathrm{R}$ and $\mathrm{S} .{ }^{23} \mathrm{Up}$ to now, this type of caged PPAPS is the most structurally complex PPAP natural product with a highly compact polycyclic ring system containing seven stereocenters, five of which are quaternary. The biosynthetic pathway of 5 was proposed as shown in Scheme 1. An intramolecular Diels-Alder reaction of $\mathrm{M}$ would form the key intermediate A, then underwent radical cyclization, tautomerization and oxidative rearomatization to give $\mathbf{5}$.

The twelve known compounds including 7 xanthones (6-12) and 5 flavonoids (13-17)were identified as 1,4,5-trihydroxyxanthone $(6),{ }^{24} 1,3,7$-trihydroxyxanthone (7), ${ }^{25}$ 1,3,5-trihydroxyxanthone (8), ${ }^{26}$ 1,5,6-trihydroxy-3-methoxyxanthone (9), ${ }^{26} \quad$ 1,3,6-trihydroxy-7methoxyxanthone (10), ${ }^{27,28} \quad$ 2,5-dihydroxy-1-methoxylxanthone (11) ${ }^{29}$ 1,3,5,6-tetrahydroxy-2-isoprenylxanthone (12), ${ }^{30}$ naringenin $(13){ }^{31}$ 6-prenyl-4',5,7-trihydroxyflavone (14), ${ }^{32}$ GB-2a (15), ${ }^{33}$ $( \pm)$-fukugetin $(\mathbf{1 6}),{ }^{34}$ volkensiflavone $(17)^{34}$ by comparison of the spectroscopic data with those reported in the literature.

\subsection{The antiproliferative activities of isolated compounds}

The antiproliferative activities of 17 compounds were evaluated on four human tumor cell lines (HepG2, A549, SGC7901, MCF7). As shown in Table 3, only compounds 1-5 and 12 could 
inhibit cancer proliferation derived from different tissues. PPAP derivatives 1-5 exhibited a stronger inhibitory effect on all four cancer cell, especially more effectively against HepG2 cell lines with $\mathrm{IC}_{50}$ values less than $10 \mu \mathrm{M}$. These results suggested that PPAPs might be responsible for the antitumor effect of $G$. xanthochymus.

\section{Conclusions}

In this study, chemical investigation on the fruits of Garcinia xanthochymus yielded five new PPAPs (1-5) and 12 known compounds. Their structures were identified by extensive spectroscopic analyses. To the best of our knowledge, this study represents the first reported account of the isolation of adamantyl PPAPs from this plant. All isolated compounds were evaluated anti-proliferative activities against four human tumor cells (HepG2, A549, SGC7901, MCF-7). As a result, PPAPs derivatives (1-5) displayed potential inhibitory activity against four human cancer cell lines with $\mathrm{IC}_{50}$ values of 5.16-16.45 $\mu \mathrm{M}$. These data suggest that the extracts of the fruits of G. xanthochymus are potent candidates for cancer prevention.

\section{Acknowledgements}

This work was financially supported by the National Science Foundation of China (No. 31370379) and Key projects of technological innovation of Hubei province (No. 2016ACA138).

\section{References}

1 S. Karuppusamy, G. Muthuraja and K. M. Rajasekaran, Indian J. Nat. Prod. Resour., 2011, 2, 174-178.

2 B. Sharma, P. J. Handique and H. S. Devi, J. Food Sci. Technol., 2015, 52, 894-902.

3 K. U. Yilmaz, S. Ercisli, Y. Zengin, M. Sengul and E. Y. Kafkas, Food Chem., 2009, 114, 408-412.

4 S. Kumar, S. Sharma and S. K. Chattopadhyay, Fitoterapia, 2013, 89, 86-125.

5 K. Trisuwan, S. Boonyaketgoson, V. Rukachaisiriku and S. Phongpaichit, Tetrahedron Lett., 2014, 55, 3600-3602.

6 S. Baggett, P. Protiva, E. P. Mazzola, H. Yang, E. T. Ressler, M. J. Basile, I. B. Weinstein and E. J. Kennelly, J. Nat. Prod., 2005, 68, 354-360.

7 J. T. Lyles, A. Negrin, S. I. Khan, K. He and E. J. Kennelly, Planta Med., 2014, 80, 676-681.

8 R. K. Baslas and P. Kumar, Acta Cienc. Indica, Chem., 1981, 7, 31-34.

9 R. K. Baslas and P. Kumar, Curr. Sci., 1979, 48, 814-815.

10 M. Fu, H. J. Feng, Y. Chen, D. B. Wang and G. Z. Yang, Chin. J. Nat. Med., 2012, 10, 129-134.

11 M. Fu, X. Hu, J. Xu, Y. Chen and G. Z. Yang, Nat. Prod. Res. Dev., 2014, 26, 255-259.

12 P. Protiva, M. E. Hopkins, S. Baggett, H. Yang, M. Lipkin, P. R. Holt, E. J. Kennelly and I. B. Weinstein, Int. J. Cancer, 2008, 123, 687-694.
13 N. Tanaka, Y. Takaishi, Y. Shikishima, Y. Nakanishi, K. Bastow, K. H. Lee, G. Honda, M. Ito, Y. Takeda, O. K. Kodzhimatov and O. Ashurmetov, J. Nat. Prod., 2004, 67, 1870-1875.

14 L. J. Zhang, C. T. Chiou, J. J. Cheng, H. C. Huang, L. M. Yang Kuo, C. C. Liao, K. F. Bastow, K. H. Lee and Y. H. Kuo, J. Nat. Prod., 2010, 73, 557-562.

15 C. Tantapakul, W. Phakhodee, T. Ritthiwigrom, S. Cheenpracha, U. Prawat, S. Deachathai and S. Laphookhieo, J. Nat. Prod., 2012, 75, 1660-1664.

16 H. P. Pepper, H. C. Lam, W. M. Bloch and J. H. George, Org. Lett., 2012, 14, 5162-5164.

17 R. Ciochina and R. B. Grossman, Chem. Rev., 2006, 106, 3963-3986.

18 L. H. Hu and K. Y. Sim, Tetrahedron Lett., 1998, 39, 79998002.

19 H. Zhu, C. Chen, J. Yang, X. Li, J. Liu, B. Sun, S. Huang, D. Li, G. Yao, Z. Luo, Y. Li, J. Zhang, Y. Xue and Y. Zhang, Org. Lett., 2014, 16, 6322-6325.

20 G. E. Henry, H. Jacobs, S. McLean, W. F. Reynolds and J. Yang, Tetrahedron Lett., 1995, 36, 4575-4578.

21 O. Thoison, D. D. Cuong, A. Gramain, A. Chiaroni, N. V. Hung and T. Séveneta, Tetrahedron, 2005, 61, 85298535.

22 J. Zhang, J. Yang, Y. Liao, X. Yang, J. Ma, Q. Xiao, L. Yang and G. Xu, Org. Lett., 2014, 16, 4912-4915.

23 Y. Liao, S. Yang, X. Li, X. Yang and G. Xu, Sci. China: Chem., 2016, 59, 1216-1223.

24 M. Iin uma, H. Tosa, T. Tanaka, F. Asai and R. Shimano, Phytochemistry, 1995, 38, 247-249.

25 C. N. Lin, M. I. Chung, S. J. Liou, T. H. Lee and J. P. Wang, J. Pharm. Pharmacol., 1996, 48, 532-538.

26 W. Li, Y. N. Sun, X. T. Yan, S. Y. Yang, C. W. Choi, J. W. Hyun, H. K. Kang, K. Y. Paek and Y. H. Kim, Food Sci. Biotechnol., 2013, 22, 945-949.

27 M. Linuma, H. Tosa, T. Ito, T. Tanaka and S. Riswan, Chem. Pharm. Bull., 1996, 44, 1744-1747.

28 H. Tosa, M. Linuma, K. I. Murakami, T. Ito, T. Tanaka, V. Chelladurai and S. Riswan, Phytochemistry, 1997, 45, 133-136.

29 H. Minami, E. Takahashi, M. Kodama and Y. Fukuyama, Phytochemistry, 1996, 41, 629-633.

30 K. Panthong, W. Pongcharoen, S. Phongpaichit and W. C. Taylor, Phytochemistry, 2006, 67, 999-1004.

31 W. Gao, L. Yang, H. H. Li, C. Q. Liu, S. J. Liu and J. M. Hu, Mod. Chin. Med., 2015, 17, 311-314.

32 S. J. Lee, A. R. Wood, C. G. A. Maier, R. A. Dixon and T. J. Mabry, Phytochemistry, 1998, 49, 2573-2577.

33 B. M. Abegaz, B. T. Ngadjui, E. Dongo and H. Tamboue, Phytochemistry, 1998, 49, 1147-1150.

34 R. S. Compagnone, A. C. Suarez, S. G. Leitao and F. D. Monache, Rev. Bras. Farmacogn., 2008, 18, 6-10. 